\title{
The Significance of Frontal Intermittent Rhythmic Delta Activity in Children
}

\author{
Shay Menascu, Ismail Mohamed, Shany M. Tshechmer, Manohar Shroff, \\ Miguel A. Cortez
}

\begin{abstract}
Objective: To determine the correlation between frontal intermittent rhythmic delta activity (FIRDA) and the clinical and radiological correlates in children. Methods: Retrospective review of the EEG and imaging studies of 37 children with documented FIRDA. Results: FIRDA was associated with multiple neurological conditions and not necessarily with midline lesions. Patients with abnormal neurological exam had a longer FIRDA duration (average 9.5 seconds) compared to children with no reported abnormal examination (average of 6.5 seconds). FIRDA ranged from 2 to $2.5 \mathrm{~Hz}$ $(n=15), 3 \mathrm{~Hz}(n=17)$ and from 1.5 to $3 \mathrm{~Hz}(n=5)$ and there was a significant association between the duration of FIRDA and abnormal laboratory tests ( $p .<0.05$, Student's T test). Conclusion: FIRDA was not correlated with midline brain lesions in children. FIRDA may be a non specific oscillation of an unhealthy pediatric brain with or without seizures.
\end{abstract}

RÉSUMÉ: La signification de l'activité delta rythmique intermittente frontale chez les enfants. Objectif : Le but de l'étude était de déterminer la corrélation entre l'activité delta rythmique intermittente frontale (ADRIF) et les constatations cliniques et radiologiques chez les enfants. Méthodes : Nous avons procédé à une revue rétrospective des EEG et de l'imagerie chez 37 enfants présentant une ADRIF documentée. Résultats : L'ADRIF était associée à plusieurs maladies neurologiques, pas nécessairement des atteintes de structures médianes. Les patients dont l'examen neurologique était anormal présentaient une ADRIF dont la durée était plus longue (moyenne de 9,5 secondes) comparée à celle d'enfants dont l'examen neurologique était normal (moyenne de 6,5 secondes). L'ADRIF était de 2 à $2,5 \mathrm{~Hz}(\mathrm{n}=15)$, de $3 \mathrm{~Hz}(\mathrm{n}=17)$ et de 1,5 à $3 \mathrm{~Hz}(\mathrm{n}=5)$ et il y avait une association significative entre la durée de l'ADRIF et les examens de laboratoire anormaux ( $p<0,05$, test $t$ de Student). Conclusion : L'ADRIF n'était pas corrélée à des lésions cérébrales médianes chez les enfants. Il est possible que l'ADRIF soit une oscillation non spécifique d'un cerveau d'enfant malade, avec ou sans crises convulsives.

Can. J. Neurol. Sci. 2010; 37: 656-661

Frontal intermittent rhythmic delta activity (FIRDA) is a transient electroencephalographic (EEG) finding with unknown pathophysiological significance. Frontal intermittent delta activity has been reported in different pathological conditions of the central nervous system. Initially FIRDA was described by Cobb in 1945, who attributed its occurrence to a midline deep lesion ${ }^{1}$. Frontal intermittent delta activity is known to occur in children as well, however, there are few studies on the clinical significance of FIRDA and the specific pathologies in the pediatric population. The last reported article on this EEG finding comes from Watemberg and his colleagues ${ }^{2}$ who collected 20 children that had presented with FIRDA on their EEGs from three pediatric centers, and analyzed their medical files including causes for admissions, laboratory findings and any report of abnormal brain imaging or cognitive status. It was concluded that FIRDA had longer duration in children with impaired cognition, unlike children with epilepsy whose FIRDA was clearly independent from the epileptiform discharges. In this study, FIRDA was a rare finding that did not correlate with any specific brain lesion or encephalopathy and tends to be during the wakened state. Fariello reported FIRDA in metabolic encephalopathy (22\%) and structural pathology (78\%) and Hooshmand also reported FIRDA in metabolic encephalopathies, deep midline lesions and hemispheric lesions ${ }^{3,4}$.

From the Division of Neurology (SM, MAC), Department of Diagnostic Imaging (MS), Department of Pediatrics (MS, MAC), The Hospital for Sick Children, University of Toronto, Toronto, Ontario; Department of Pediatrics (IM), University of Calgary, Calgary, Alberta, Canada: Pediatric Neurology Unit (SM), Safra Children's Hospital, TelHasomer; Department of Behavioral Sciences (SMT), Ben-Gurion University of the Negev, Beer-Sheva, Israel.

Received October 30, 2008. Final Revisions Submitted March 26, 2010. Correspondence to: Miguel A. Cortez, Division of Neurology, 555 University Avenue Toronto, Ontario, M5G 1X8, Canada. 
Other pathologies associated with FIRDA include brain tumors, pituitary adenomas, cerebral ischemic lesions, hydrocephalus, dementia, acute confusional state, migraine, increased intracranial pressure and infantile neuroaxonal dystrophy ${ }^{5-8}$. Acute metabolic derangements, including severe uremia and liver failure, were reported in up to $22 \%$ of adults with FIRDA $^{9}$, and posterior fossa tumors in up to $10 \%$ of patients with slow waves ${ }^{10}$. Frontal intermittent delta activity was also reported in approximately $40 \%$ of patients with metastatic brain tumors and $25 \%$ of patients with primary brain gliomas. Their computed tomography (CT) scans, however, did not show a significant correlation between the localization, depth and size of the tumors and the concomitant occurrence of FIRDA $^{1,10,11}$. Most of these previous reports on FIRDA were from adult patients. Therefore, the aim of this study was to determine the correlation between FIRDA and the clinical and radiological findings, in the context of the scarce literature on FIRDA in children.

\section{METHODS}

\section{Electroencephalography (EEG)}

The EEG recordings from the computerized pediatric EEG data base at the Hospital for Sick Children in Toronto were searched for the abbreviation of FIRDA and for the term frontal intermittent rhythmic delta activity, from January 1999 to December 2006. All EEG recordings with reported FIRDA were reviewed by two board certified neurophysiologists who were blinded to the patient population data, except for age and gender. Frontal intermittent delta rhythmic activity was defined as intermittent monomorphic rhythmic delta activity with maximal amplitude over the frontal regions, ranging from $1.5-3 \mathrm{~Hz} / \mathrm{sec}$ and with an amplitude of 50 to $200 \mu \mathrm{V}$.

All EEG's were obtained using the international 10-20 system of electrode placement, for a minimum of 20 minutes duration. We included only patients between 1-18 years old, with a complete clinical file and neuroimaging investigations at the Hospital for Sick Children in Toronto. We excluded patients that were sedated prior or during the EEG recording or were admitted due to drug intoxication, status epilepticus or had electrographic or clinical seizures during the recordings. Patient data included reason for testing, background activity and reactivity, presence of frontal slow activity including frequency and duration, or the presence of any other slow wave activity in both hemispheres. Also the type and localization of epileptic activity and the clinical status of the patient during the appearance of the FIRDA were analyzed.

In addition, the hospital records were reviewed to enter the demographic data such as age, gender, perinatal and postnatal history, possible previous central nervous system insults, metabolic or genetic syndrome, the full neurodevelopmental assessment and current physical status of each patient.

All possible chronic illnesses including history for any epileptic syndromes were recorded. We also recorded any reports of illness around the admission time or on admission. All laboratory tests that each patient had during admission such as blood chemistry, drugs levels, liver function, lactate, ammonia, and cerebrospinal fluid analysis, were also collected.

\section{RESULTS}

Out of 14,000 EEG recordings performed between 1999 and 2006, FIRDA was reported in only 45 children. Only 37 cases with FIRDA were identified by the blinded EEG interpretation and no patients were excluded because of lack of imaging. Of the 37 cases, 20 were female $(\mathrm{F})$ and 17 male $(\mathrm{M})$ and their age ranged from 1.8 to 18 years, (mean age 11 years $\mathrm{SD} \pm 7$ years) (Figure 1).

The full medical history including imaging was available to us for all 37 children. Pregnancy, birth and development were normal in 26 children $(70.2 \%)(21 \mathrm{~F}$ and $5 \mathrm{M})$. Only five children were born prematurely and two had a complex difficult delivery. The EEG was requested for suspected seizure activity in 56.8\% of the 37 cases. Other indications for EEG requisition (43.2\%) included encephalopathy associated with infection such as pneumonia or viral meningitis $(21.6 \%)$, non specified metabolic conditions $(8.1 \%)$, headaches $(5.4 \%)$, not specified $(5.4 \%)$ and limb tremors $(2.7 \%)$. (Table 1$)$.

\section{EEG Findings}

Twenty-five children (67.6\%) had one EEG recording during admission where FIRDA was reported (Figure 2). Twelve children $(32.4 \%)$ had more than one EEG recording during the same admission and FIRDA was reported in at least one of the recordings. Frontal intermittent delta activity occurred during wakefulness in 31 patients $(83.8 \%)$, during drowsiness in five $(13.5 \%)$ and in a child with stupor state $(2.7 \%)$. The majority of the children $(74 \%)$ had at least one abnormal neurological exam prior to their EEG recording. Of these children, $35 \%$ were suspected to be in post ictal state, $21 \%$ had asymmetric hyper or hypo patellar or biceps reflexes, $10 \%$ had transient mono or hemiparesis and $8 \%$ were found to have abnormal eye movements.

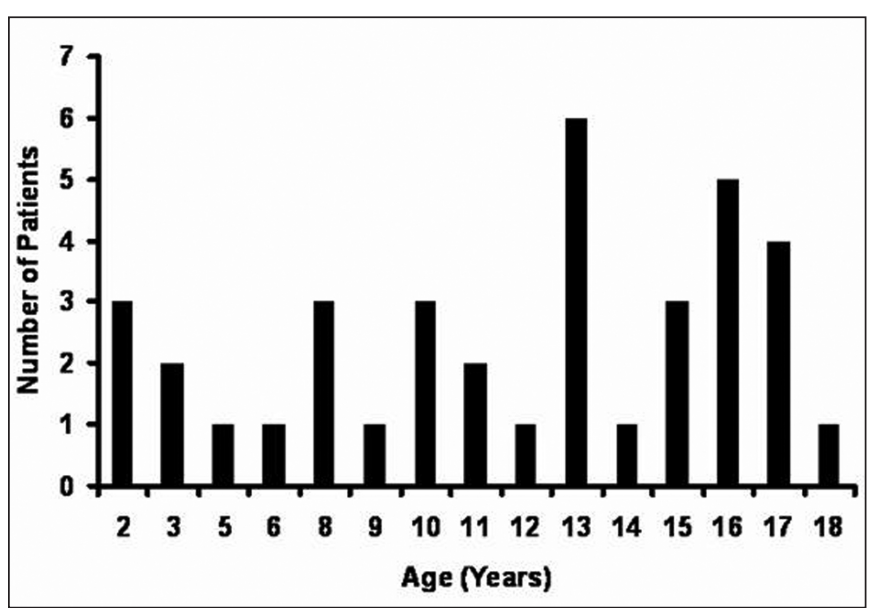

Figure 1: The age of the patients with documented FIRDA that were included in the study ranged from 2 to 18 years-of-age (Mean $+S D=$ $11 \pm 7)$. There were 14 children (38\%) under 10 years and 23 children (62\%) from 11 to 18 years-of-age. 
Table 1: Indication for EEG in patients with FIRDA

\begin{tabular}{lcc}
\hline Reason for EEG requisition & No. & \% \\
Seizures & 21 & 57 \\
Encephalopathy & 8 & 21.6 \\
Metabolic conditions & 3 & 8.1 \\
Not specific & 2 & 5.4 \\
Headaches & 2 & 5.4 \\
Limb tremor & 1 & 2.7 \\
\hline
\end{tabular}

The duration of FIRDA ranged from 3 to 4 seconds in 7 children (19\%), from 5 to 8 seconds in $10(27 \%)$, and from 9 to 11 seconds in 20 children $(54 \%)$. Patients with abnormal neurological exam had a relatively longer FIRDA duration (Mean $\pm \mathrm{SD}=9.5 \pm 2.23$ seconds), compared to children who did not have abnormal neurological examination (Mean $\pm \mathrm{SD}=6.5 \pm$ 1.97 seconds), $95 \%$ confidence interval of the difference $=-1.176$ to $0.3176, t=1327,34$ degrees of freedom, Two-tailed $\mathrm{P}=0.2653$, therefore not considered significant. The frequency of FIRDA varied between 2 to $2.5 \mathrm{~Hz}$ in 15 cases $(40.5 \%), 3 \mathrm{~Hz}$ in 17 cases (45.9\%) and from 1.5 to $3 \mathrm{~Hz}$ in 5 cases (13.6\%). Twenty-two children $(59.4 \%)$ (14 F and $8 \mathrm{M}$ ) had abnormal laboratory tests prior to their EEG recording, including mild elevation of liver function assessments in 13 cases, mild elevation of serum lactate in seven cases and hyponatremia (around $130 \mathrm{mml} / \mathrm{dl}$ ) in two.

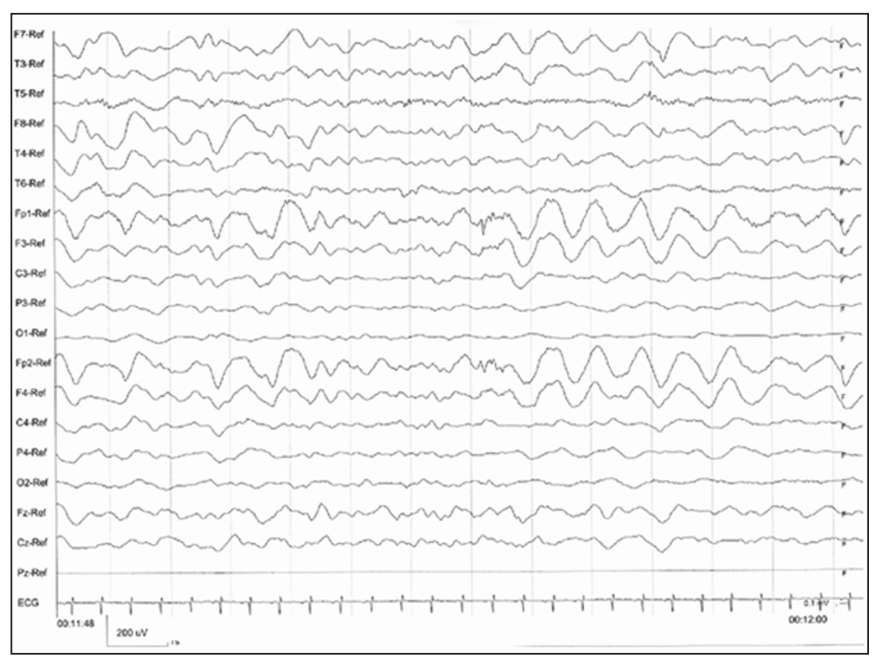

Figure 2: A characteristic Frontal Intermittent Rhythmic Delta Activity (FIRDA) is depicted with a $C z$ reference montage. FIRDA consisted of an intermittent monomorphic rhythmic slow oscillation with maximal amplitude over the bilateral frontal head regions, lasting up to four seconds. The cortical distribution and the frequency of 1.5-2.5 Hz and the medium to high $200 \mu \mathrm{V}$ amplitude are both consistent with FIRDA. Time/ Voltage scale indicate 1 second and $200 \mu \mathrm{V}$.
There was a statistically significant association between patients with abnormal laboratory findings and a longer duration of FIRDA ( $p<0.05$, Student's t Test).

The EEG background activity was abnormal in 30 children (81\%). Twelve of these children (10 F and $2 \mathrm{M}$, age range 3-16.5 years, mean age 8.8 years) had 4 to $6 \mathrm{~Hz}$ activity compared to eight girls and five boys (age range 4.5-16 years, mean age 11 years) that had $5-7 \mathrm{~Hz}$ activity. Five female patients had diffuse theta activity (age range 6-13 years, mean age 9.5 years). The duration of FIRDA was not different between patients from 1 to 10 years and patients from 11 to 18 years of age $(p .=0.46$, Student's t Test). More than half of the patients (57\%) did not

Table 2: Number of patients on Antiepileptic Drugs

\begin{tabular}{lcc}
\hline AED & Number of Patients & Percent (\%) \\
KEP & 5 & 13.5 \\
GVG & 4 & 10.8 \\
PHY & 2 & 5.4 \\
CBZ & 3 & 8.1 \\
TOP & 5 & 13.5 \\
VPA & 12 & 32.4 \\
LAM & 6 & 16.2 \\
Total & 37 & 100.0 \\
\hline
\end{tabular}

$\mathrm{AED}=$ Antiepileptic Drugs; KEP $=$ Keppra; $\mathrm{GVG}=$ Vigabatrin; $\mathrm{PHY}=$ Phenytoin; $\mathrm{CBZ}=$ Carbamazepine; TOP=Topiramate; VPA=Valproic Acid; LAM=Lamotrigine. Some of these medications were used in combination of two or more AED's.

have any epileptiform activity during the recordings. The remaining patients had either temporal $(20 \%)$, occipital $(12 \%)$, central $(8 \%)$ or parietal $(3 \%)$ epileptiform discharges. No significant correlation between FIRDA and the localization of the epileptic discharges was found.

The comparison between patients with cognitive abnormalities ( $9 \mathrm{~F}$ and $7 \mathrm{M}$ ) and those with intact cognitive function (11 F and $10 \mathrm{M}$ ) showed no significant association with either the presence of epileptic discharges or the duration of FIRDA. More than $65 \%$ of the patients were on different types of medications and $45 \%$ on antiepileptic drugs during the EEG recordings (Table 2).

\section{Brain Imaging Studies}

All children had at least one neuroimaging study and $34 \%$ had more than one study. Eleven children had brain CT, 16 children had brain magnetic resonance imaging (MRI) and ten children had both CT and brain MRI studies. Twenty children (55\%) had normal imaging and 17 children had abnormal findings on their 
brain CT, MRI or both as follows: six had subcortical and gray matter lesions, five had cortical and white matter lesions and seven had both. No significant statistical correlation was found between any abnormal imaging and FIRDA duration.

There were only two cases with distinct midline abnormalities with FIRDA in this group of patients. The first, an eight-year-old boy presenting with rapidly progressive encephalopathy with MRI T2 axial (a) and FLAIR axial (b) images that show bilateral diffuse increase in signal in the thalami, basal ganglia and insular cortex in keeping with the diagnosis of encephalitis (Figure 3) had FIRDA lasting up to four seconds in duration. The second case, a premature child born at 36 weeks of gestational age, with dysmorphic features had an MRI scan at two weeks-of-age showing dysgenesis of the corpus callosum (Figure 4). Frontal intermittent delta activity like epochs lasting up to eight seconds in duration were recorded at the age of 14 months in this child. The sagittal image shows thin posterior body of the corpus callosum and absence of the splenium, along with inferior vermian hypoplasia. The lateral ventricles are dysplastic and parallel as seen on the coronal and axial images. In both cases the EEG was reactive to external stimulation.

\section{DiscuSSION}

Historically, FIRDA was reported in association with different neurological conditions. Frontal intermittent delta activity in the waking adult was first attributed to epithalamic tumors and subsequently identified in posterior fossa and deep midline pathologies ${ }^{1,10,12}$. In adults, FIRDA was also associated with metabolic conditions and diffuse or focal intracranial diseases, and was previously accepted as a sign of pathological processes in the central nervous system ${ }^{13,14}$.

In our study most patients had an initial abnormal neurological exam and up to $35 \%$ had altered mental status and the duration of FIRDA was significantly increased in patients with abnormal laboratory tests. Abnormal laboratory findings (37\%) also correlated with longer duration of FIRDA. Watemberg reported five out of 22 children with FIRDA with concurrent laboratory tests and only one of them had mildly elevated serum aspartate and alanine transaminase but these laboratory findings did not correlate to a prolonged FIRDA ${ }^{2}$. We deduce that metabolic abnormalities have received a modest consideration in the existing literature in the pediatric field and suggest that the occurrence of FIRDA in children represents an abnormal electrical oscillation that is not necessarily dependent on a specific midline brain lesion ${ }^{1,12}$ and, instead, may be consistent with a systemic metabolic condition in the developing brain.

Watemberg reported in 2003 a correlation between impaired cognition and longer bursts of FIRDA in children ${ }^{2}$. In our study, we could not replicate this observation and in addition, the duration of FIRDA did not correlate to the age of our patients or to the frequency of their posterior dominant background activity. Our children who showed FIRDA did not require a longer hospital stay (average 5.2 days) compared to the average admission time for children admitted with other neurological diseases during this time, suggesting the finding of FIRDA may not adversely affect the short term outcome of these patients. We acknowledge that the lack of long term follow-up data in our study constitutes a limitation of our study.

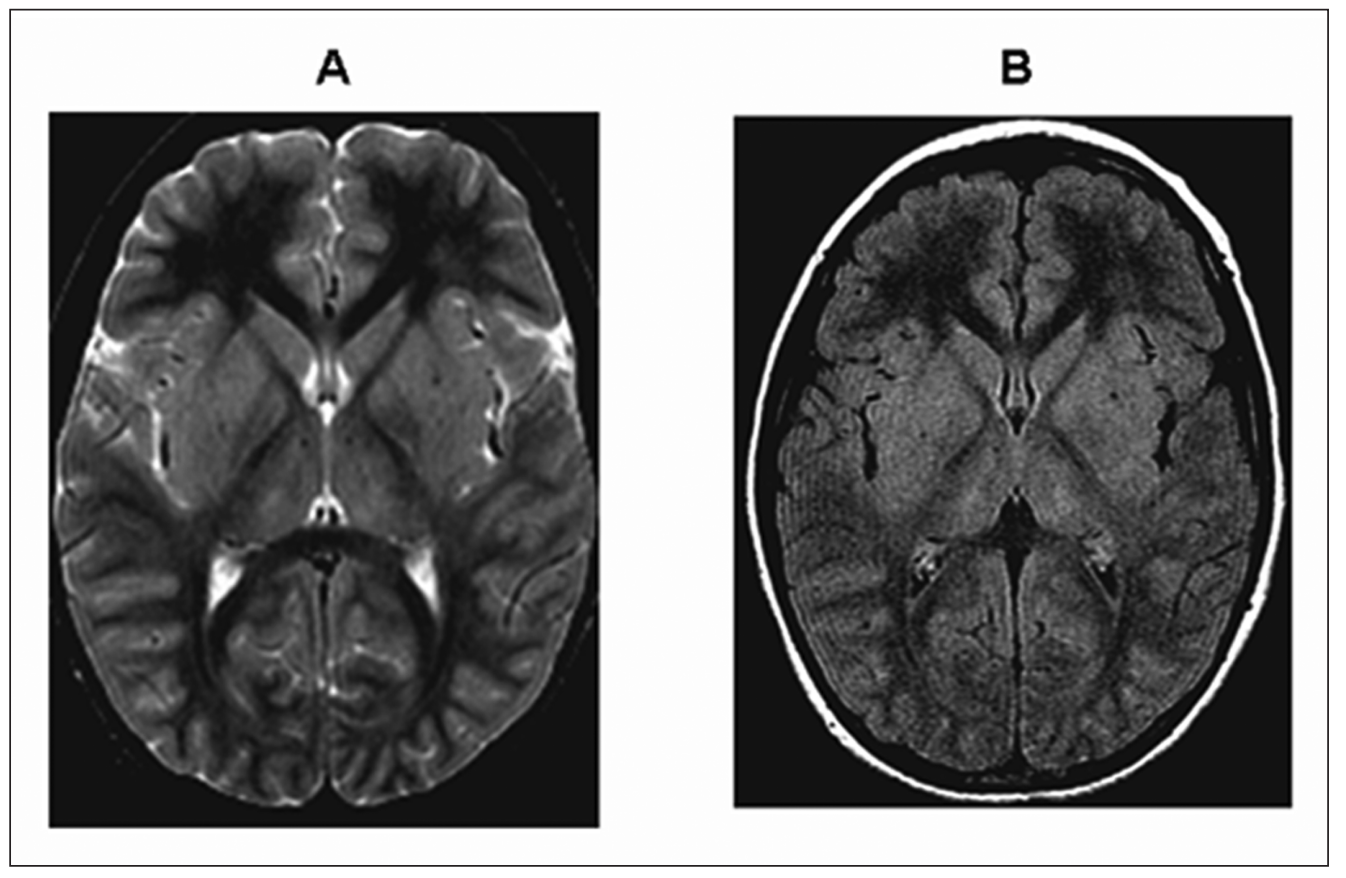

Figure 3: MRI in an eight-year-old boy presenting with rapidly progressive encephalophathy: T2 axial (A) and FLAIR axial (B) images show bilateral diffuse increase in signal in the thalami, basal ganglia and insular cortex in keeping with the diagnosis of encephalitis. 


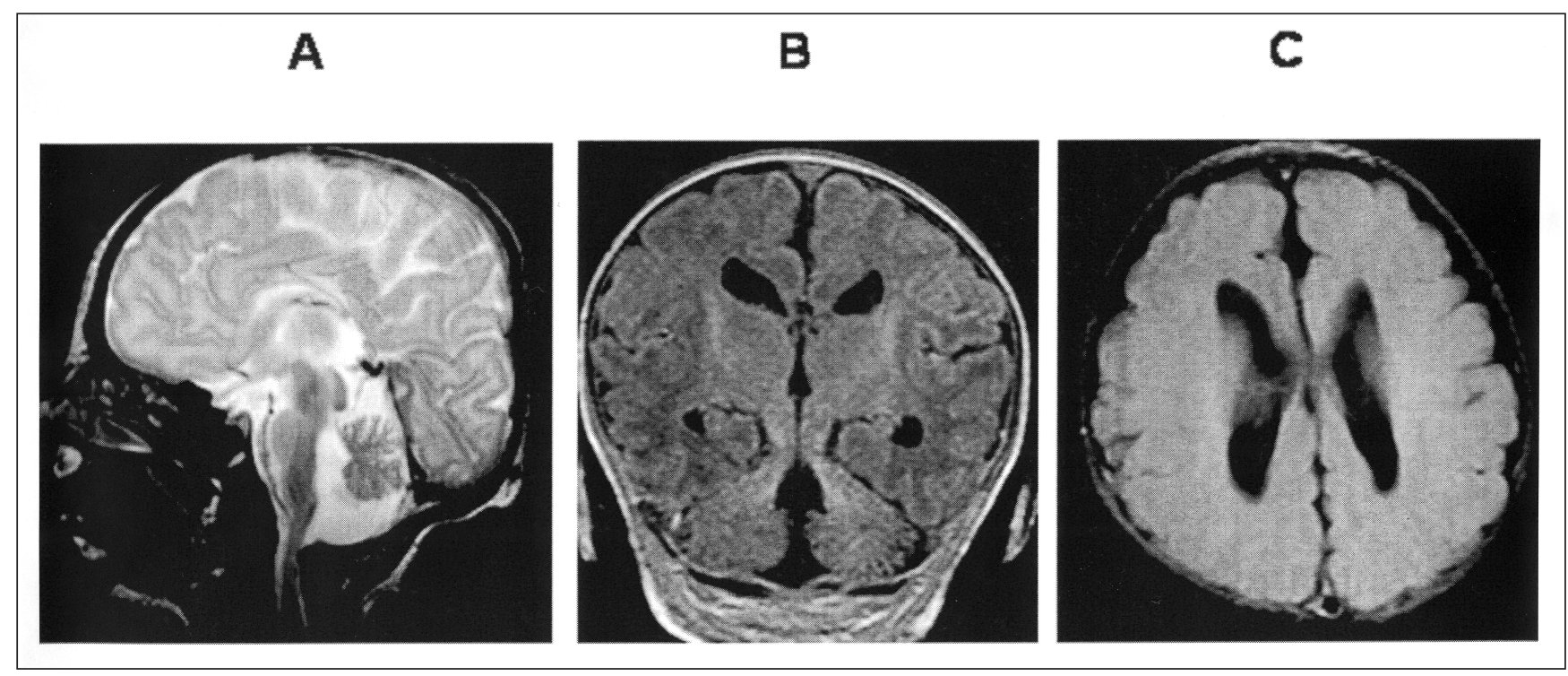

Figure 4: MRI in a two-week-old premature child born at 36 weeks of gestational age, with dysmorphic features: Sagittal T1 (A), coronal T2 (B) and axial FLAIR $(C)$ images show dysgenesis of the corpus callosum. The sagittal image shows thin posterior body of the corpus callosum and absence of the splenium, along with inferior vermian hypoplasia. The lateral ventricles are dysplastic and parallel as seen on the coronal and axial images.

The MRI in some patients with FIRDA showed diffuse cerebral structural lesions, periventricular white matter disease and cortical atrophy of varying severity ${ }^{2}$. The CT findings in patients with FIRDA assessing the possibility of shifting of midline structures showed no correlation between mass effect and the prevalence of FIRDA. Deep midline lesions were present only in a minority of patients with FIRDA ${ }^{15}$. Different reports have speculated that FIRDA may represent projections from deep subcortical midline structures or may reflect pathological signs of gray matter disease ${ }^{14}$. We confirmed with our series that the association between deep midline or infratentorial lesions and FIRDA was also rare.

A study done by Scollo-Lavizzari assessed the correlation between CT findings in adults, and the incidence of FIRDA by looking at shifting of midline structures as a sign of mass effect that can lead to FIRDA formation, but did not find such a mass in 23 of 26 patients reported with FIRDA ${ }^{15}$. Fariello studied 80 adult patients with FIRDA and reported focal lesions in $44 \%$, diffuse structural changes in $34 \%$ and no pathology in $22 \%{ }^{3,16}$. A recent study ${ }^{17}$ on MRI correlation and FIRDA in 27 pediatric patients, diffuse periventricular white matter and cortical atrophy, were the most prevalent findings reported. In our series, all children had at least one neuroimaging study and $34 \%$ had more than one study. Only 17 out of 37 children had a pathological finding that was detected on imaging. In these cases, subcortical and gray matter lesions $(24 \%)$ were the most common findings and there was no significant correlation between abnormal imaging and FIRDA duration, which is consistent with the Watemberg study ${ }^{2}$.

There are only a few reports on the prevalence of FIRDA in pediatric population. Frontal intermittent delta activity was detected in 15 out of 32 patients with tuberculosis meningitis (age range between 8 to 62 years) and in 11 patients (age range
8 to 23 years) during acute migraine episodes ${ }^{18,19}$. These patients were encephalopathic during these migraine attacks and their EEG's showed FIRDA with slow background activity. Frontal intermittent delta activity is associated with encephalopathy in adults, however acute encephalopathy is rarely reported in correlation with FIRDA in children ${ }^{12,15,18,19}$. In our retrospective study we are cautious to establish such a correlation because more than $65 \%$ of the patients were on different types of medications and $45 \%$ on antiepileptic drugs at the time of the EEG recordings. Therefore, a prospective study would be warranted to determine the correlation between FIRDA and acute encephalopathy ${ }^{20}$ and furthermore other correlations between FIRDA and infections, inflammatory or metabolic abnormalities for which an EEG was indicated in these children with FIRDA.

The EEG findings in children who had been mentally challenged were reported with slow background activity and a higher percentage of paroxysmal discharges than age matched cognitively normal patients ${ }^{21,22}$. Watemberg reported that FIRDA duration appeared to correlate to neurocognitive status in children, as those with impaired cognition had longer FIRDA duration. He speculated that longer duration of FIRDA could be a sign of frontal lobe dysfunction in mentally challenged children $^{2}$. Due to the retrospective character of our study, we could not directly evaluate the intellectual functioning of our patients. Our data however, did not show any significant correlation between patients reported to have cognitive impairments and either the longer duration of FIRDA or the slower background activity.

Riviello and Foley suggested that FIRDA is an epileptiform pattern in children based on the fact that up to $85 \%$ of their patients with FIRDA had seizures ${ }^{23}$ and another recent study ${ }^{24}$ reported FIRDA in up to $2 \%$ of the patients found to have 
different types of epilepsy. Those patients with FIRDA of the frequency of $3 \mathrm{~Hz}$ were younger and had a higher incidence of idiopathic generalized epilepsy compared to patients that showed a FIRDA frequency between 1.5 to $2.5 \mathrm{~Hz}$ who more often had partial epilepsy ${ }^{25}$. In our study, only $43 \%$ of these children had epileptiform activity on their EEG's, either unilateral or bilateral. We did not find any significant correlation between the type and the focal location of the epileptiform discharges and the FIRDA duration. Although FIRDA was often associated with epileptiform discharges in our waking EEG recordings, we could not conclude that FIRDA was epileptogenic in nature. This is another point of agreement with Watemberg's study; however, it is worth mentioning that none of the EEG recordings included in our study were performed during clinical seizures or following status epilepticus ${ }^{2}$.

More than $60 \%$ of the patients in our study were on different types of medications and $45 \%$ on antiepileptic drugs during the EEG recordings [Table 2]. We controlled for the possible drug effects and found no correlation between any specific drug and the occurrence of FIRDA and its duration. This is consistent with other studies that also failed to find any association between FIRDA and any particular chronic medication intake ${ }^{26}$.

\section{Conclusions}

To date, our retrospective study involves the largest group of children with FIRDA, excluding all possible confounding factors such as sedation, drug intoxication and ongoing ictal conditions. The occurrence of FIRDA in our Hospital was extremely low as we found only 37 cases that met our criteria, over a period of seven years. The mechanisms involved in the generation of FIRDA in children remain to be determined; therefore, we need to be cautious with the interpretation of FIRDA, especially that of longer duration and linked to abnormal laboratory findings. It is conceivable that FIRDA represents an electrographic oscillation from an unhealthy developing brain, likely under the throes of an underlying systemic metabolic condition, hence not specific to any particular midline focal lesion or an epileptic condition.

\section{ACKNOWLEDGMenTS}

The authors thank Marilyn McLaughlin for assistance in preparation of the manuscript and thank the editor for his criticisms and suggestions which helped improve the text. We confirm that we have read the Journal's position on issues involved in ethical publication and affirm that this report is consistent with those guidelines. None of the authors has any conflict of interest to disclose.

\section{REFERENCES}

1. Cobb WA. Rhythmic slow discharges in the electroencephalogram. J Neurol Neurosurg Psychiatry. 1945;8:65.

2. Watemberg N, Gandelman R, Neufeld MY, Ginsberg M, LermanSagie T, Kramer U. Clinical correlates of frontal intermittent rhythmic delta activity in children. J Child Neurol. 2003;18: $525-9$.

3. Fariello RG, Orrison W, Blanco G, Reyes PF. Neuroradiological correlates of frontally predominant intermittent rhythmic deltaactivity. Electroencephalogr Clin Neurophysiol. 1982;54: 194-202.

4. Hooshmand $\mathrm{H}$. The clinical significance of frontal intermittent rhythmic delta activity (FIRDA). Clin Electroencephalogr. 1983; 14:135-7.
5. Van der Drift JH, Magnus O. The value of the EEG in the differential diagnosis of cases with cerebral lesion. Electroencephalogr Clin Neurophysiol.1959;11:733-46.

6. Pietrini V, Terzano MG, D'Andrea G, Parrino L, Cananzi AR, Ferro-Milone F. Acute confusional migraine: clinical and electro-encephalography aspect. Cephalagia. 1987;7:29-37.

7. Kameda K, Itoh N, Nakayama H, Kato Y, Ihda S. Frontal intermittent rhythmic delta activity in pituitary adenoma. Clin Electroencephalogr. 1995;26:173-9.

8. Mutoh K, Okuno T, Ito M, Fujii T, Mikawa H. Continuous generalized, high voltage fast activity and FIRDA in two children. Clin Electroencephalogr. 1992;13:68-71.

9. Bagchi BK, Kooi KA, Selving BT, Calhoun HD. Subtentorial tumors and other lesions: An electroencephalographic study of 121 cases. Electroencephalogr Clin Neurophysiol. 1961;13: 180-92.

10. Daly D, Whelan JL, Bickford RG, MacCarty CS. The electroencephalogram in cases of tumors of posterior fossa and third ventricle. Electroencephalogr Clin Neurophysiol. 1953;5: 203-16.

11. Schaul N, Lueders H, Sachdev K. Generalized bilateral synchronous bursts of slow waves in EEG. Arch Neurol. 1981:38:690-2.

12. Stam CJ, Pritchard WS. Dynamics underlying rhythmic and nonrhythmic variants of abnormal, waking delta activity. Int $\mathbf{J}$ Psychophysiol. 1999;34:5-20.

13. Faure J, Droogleever-Fortuyn J, Gastaut H, Larramendi L, Martin $\mathrm{P}$, Passouant $\mathrm{P}$, et al. De la genese et de la significacion des rhythms recueillis a distance dans les cas de tumeurs cerebrales. Electroencephalogr Clin Neurophysiol. 1951;3:429-34.

14. Gloor P, Ball G, Schaul N. Brain lesions that produce delta waves in the EEG. Neurology. 1977;27:326-33.

15. Scollo-Lavizzari G, Matthis H. Frontal intermittent rhythmic delta activity: a comparative study of EEG and CT scan findings. Eur Neurol. 1981;20:1-3.

16. Neufeld MY, Chistik V, Chapman J, Korczyn AD. Intermittent rhythmic delta activity (IRDA) morphology cannot distinguish between focal and diffuse brain disturbances. J Neurol Sci. 1999; 164:56-9.

17. Watemberg N, Towne AR. Radiological correlates of frontal intermittent rhythmic delta activity. Epilepsia. 1997;38 Suppl $4 ; 119$

18. Wasler H, Isler H. Frontal intermittent rhythmic delta activity. Impairment of consciousness and migraine. Headache. 1982;22; 74-80.

19. Kalita J, Misra UK. EEG changes in tuberculous meningitis: a clinicoradiological correlation. Electroencephalogr Clin Neurophysiol. 1998;107:39-43.

20. Watemberg N, Alehan F, Dabby R, Lerman-Sagie T, Pavot P, Towne A. Clinical and radiologic correlates of frontal intermittent rhythmic delta activity. J Clin Neurophysiol. 2002;19:535-9.

21. Gasser T, Möcks J, Lenard HG, Bächer P, Verleger R. The EEG of mildly retarded children: developmental, classificatory and topographic aspects. Electroencephalogr Clin Neurophysiol. $1983 ; 55: 131-44$.

22. Martín-Loeches M, Muňos-Ruata J, MartÍnez-Lebrusant L, GómezJarabo G. Electrophysiology and intelligence: the electrophysiology of intellectual functions in intellectual disability. $\mathrm{J}$ Intellec Disabil Res. 2001;45:63-75.

23. Riviello JJ Jr, Foley CM. The epileptiform significance of intermittent rhythmic delta activity in children. J Child Neurol. 1992;7:156-60

24. Woolridge D, Stefanelli M, Hoppe B. Susac syndrome with frontal intermittent rhythmic delta activity (FIRDA). Can J Neurol Sci. 2006;33:403-6.

25. Kubota F, Ohnishi N. Study on FIRDA and $3 \mathrm{~Hz}$ rhythmic slow wave bursts occurring in the frontal area of epileptic patients. Clin Electroencephalogr. 1997;28:112-6.

26. Calzetti S, Bortone E, Negrotti A, Zinno L, Mancia D. Frontal intermittent rhythmic delta activity (FIRDA) in patients with dementia with Lewy bodies: a diagnostic tool? Neurol Sci. 2002;23 Supp 2:S65-6. 